\title{
Plasmons in strongly coupled shock-compressed matter
}

P. Neumayer, C. Fortmann, T. Doppner, P. Davis, R. W. Falcone, A. L. Kritcher, O. L. Landen, H. J. Lee, R. W. Lee, C. Niemann, S. Le Pape, S. H. Glenzer

May 13, 2010

Physical Review Letters 
This document was prepared as an account of work sponsored by an agency of the United States government. Neither the United States government nor Lawrence Livermore National Security, LLC, nor any of their employees makes any warranty, expressed or implied, or assumes any legal liability or responsibility for the accuracy, completeness, or usefulness of any information, apparatus, product, or process disclosed, or represents that its use would not infringe privately owned rights. Reference herein to any specific commercial product, process, or service by trade name, trademark, manufacturer, or otherwise does not necessarily constitute or imply its endorsement, recommendation, or favoring by the United States government or Lawrence Livermore National Security, LLC. The views and opinions of authors expressed herein do not necessarily state or reflect those of the United States government or Lawrence Livermore National Security, LLC, and shall not be used for advertising or product endorsement purposes. 


\title{
Plasmons in strongly coupled shock-compressed matter
}

\author{
P. Neumayer ${ }^{*}{ }^{1}$ C. Fortmann,,${ }^{1,2}$ T. Döppner, ${ }^{1}$ P. Davis,${ }^{1,3}$ R. W. Falcone,${ }^{3}$ A. L. Kritcher,${ }^{1,4}$ \\ O. L. Landen, ${ }^{1}$ H. J. Lee ${ }^{5}$ R. W. Lee,${ }^{1}$ C. Niemann,,${ }^{1}$, S. Le Pape,${ }^{1}$ and S. H. Glenzer ${ }^{1}$ \\ ${ }^{1}$ Lawrence Livermore National Laboratory, Livermore, CA 94551, USA \\ ${ }^{2}$ Department of Physics and Astronomy, University of California, Los Angeles, CA 90095, USA \\ ${ }^{3}$ Department of Physics, University of California, Berkeley, CA 94720, USA \\ ${ }^{4}$ Nuclear Engineering Department, University of California, Berkeley, CA 94720, USA \\ ${ }^{5}$ SLAC National Accelerator Laboratory, 2575 Sand Hill Rd. Menlo Park, CA 94025
}

(Dated: April 19, 2010)

\begin{abstract}
We present the first measurements of the plasmon dispersion and damping in laser shock-compressed solid matter. Petawatt laser produced $\mathrm{K}-\alpha$ radiation scatters on boron targets compressed by a 10 ns-long $400 \mathrm{~J}$ laser pulse. In the vicinity of the Fermi momentum, the scattering spectra show dispersionless, collisionally damped plasmons, indicating a strongly coupled electron liquid. These observations agree with x-ray scattering calculations that include both the Born-Mermin approximation to account for electron-ion collisional damping and local field corrections reflecting electron-electron correlations.
\end{abstract}

PACS numbers: 52.25.Os, 61.05.cf, 78.70.Ck, 52.50.Lp

Investigation of matter under shock-compression in the laboratory is an important technique to study the physics of dense matter. Modeling astrophysical objects including e.g. interiors of giant planets $[1,2]$, low mass stars and brown dwarfs $[3,4]$ requires understanding of matter at pressures exceeding 1 Mbar [5, 6] and temperatures of the order $1 \mathrm{eV}[7]$. These conditions are produced through interaction of energetic high power lasers with solid density targets. In addition, the quest of producing a burning fusion plasma in the laboratory [8] by compressing matter up to $1000 \mathrm{~g} / \mathrm{cm}^{3}$ requires accurate knowledge of the equation of state $[9,10]$, thermodynamic [11] and transport properties [12], as well as static and dynamic structure of matter under such extreme conditions.

Spetrally resolved x-ray scattering has been shown to measure the microscopic physical properties of dense matter [13]. Proof-of-principle experiments in isochorically heated beryllium [14] have observed plasmons in forward scattering geometry yielding electron density and temperature when applying the Born-Mermin theory $[15,16]$ to calculate the plasmon energy shift and damping. Subsequent measurements in shock-compressed matter have provided density data consistent with the width of Compton scattering from a dense Fermi degenerate plasma [17]. In addition, plasmon measurements were successful in determining compression and ionization in coalescing shocks [11].

In these nearly Fermi degenerate systems reached by shock-compression, electron-ion collisions solely determine the plasmon width for small momentum transfers $\mathbf{k}$, where non-collisional (Landau) damping is suppressed, [18], and the plasmon width is a direct measure of the

*present address: GSI Helmholtzzentrum für Schwerionenforschung, Planckstrasse 1, 64291 Darmstadt, Germany optical conductivity. In addition, $k$-vector resolved measurements of the plasmon resonance energy will reflect electron-electron interactions that will affect the dispersion in the vicinity of the Fermi momentum. Theoretical models for the dynamical structure factor $S(k, \omega)$, result in vastly different dispersions. The widely applied random phase approximation (RPA) as well as the Born-Mermin theory accounting for electron-ion collisions predict a roughly parabolic dispersion [19], following the Bohm-Gross approximation [20]. Plasmon dispersion data deviating from the RPA have been observed in cold metals [21-23]. In shock-compressed matter, the decrease of the plasmon dispersion due to electron-electron local field effects will occur simultaneously with electronion collisions affecting plasmon damping.

In this Letter we present the first frequency and $k$ vector resolved plasmon spectra from shock-compressed matter. Boron samples have been compressed by interaction with high energy laser pulses, resulting in conditions above solid density, electron density $n_{\mathrm{e}}=4 \times 10^{23} \mathrm{~cm}^{-3}$, at moderate temperature, $k_{\mathrm{B}} T_{\mathrm{e}}=0.2 \mathrm{eV}$. When the shock front has reached the rear surface of the target, resulting in a homogeneously compressed sample, the plasma conditions have been probed by laser produced $\mathrm{K}-\alpha$ radiation at two energies $(5 \mathrm{keV}$ and $8 \mathrm{keV})$ under various scattering angles $31^{\circ}<\theta<68^{\circ}$. The source size and acceptance angle of the spectrometer lead to an accuracy of $\pm 10 \%$ for the scattering angles. While experimental spectra in the limits of small and large $k$-vectors, provide independent measurements of the plasma conditions, we further observe a horizontal plasmon dispersion in the range of wavevectors between $k=1.7 \AA^{-1}$ and $2.0 \AA^{-1}$. These results agree with theoretical predictions for the dynamical structure factor within the extended Born-Mermin approximation (BMA). This model includes electron-electron correlations via the local field 


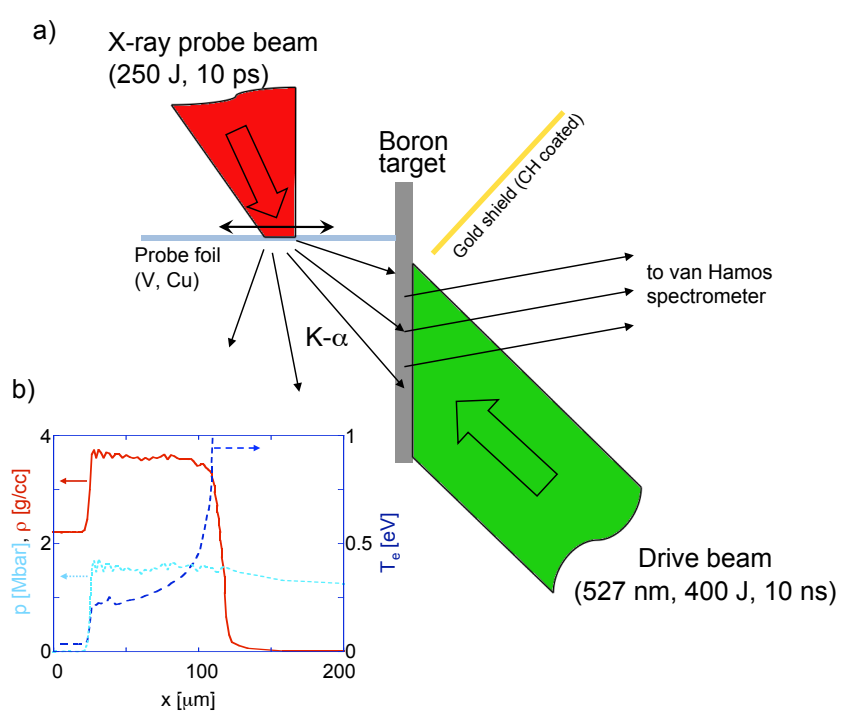

FIG. 1: (Color online) a) Schematic of the experiment showing the drive beam that compresses boron and the $\mathrm{x}$-ray probe beam that is delayed by $10 \mathrm{~ns}$ producing the $\mathrm{x}$-ray probe radiation. The probe foil material and probe laser position on the foil is varied to access a range of $k$-vectors. X-ray scattering spectra are observed with a van-Hamos spectrometer.

b) Mass density, electron temperature, and pressure as function of target depth at the end of the pump laser- target interaction $(t=10 \mathrm{~ns})$ from radiation hydrodynamic simulation (HELIOS). The irradiated surface is located at $x=200 \mu \mathrm{m}$.

correction factor (LFC) [24] and electron-ion collisions via the dynamical electron-ion collision frequency [25]. These effects account for differences of $3 \mathrm{eV}$ in plasmon width and $10 \mathrm{eV}$ in shift when compared to the RPA resulting in significant corrections for applications of x-ray Thomson scattering to plasma diagnostics.

The experiment, carried out at the Jupiter Laser Facility at Lawrence Livermore National Laboratory uses rectangular slabs of $2 \times 3 \mathrm{~mm}^{2}$ of solid boron ( $\alpha$ phase, mass density $2.46 \mathrm{~g} / \mathrm{cm}^{3}$ ), polished down to a thickness of $170 \mu \mathrm{m}$. A schematic of the experimental setup is shown in Fig. 1 a). A $2 \omega(527 \mathrm{~nm})$ drive laser laser with energy of $400 \mathrm{~J}$ compresses the boron samples in a $10 \mathrm{~ns}$-long flat top pulse. The laser radiates the target at an angle of $37.5^{\circ}$ to the target normal with a homogeneous intensity profile applying a $700 \mu \mathrm{m}$ continuous phase plate providing for a planar shock front to propagate into the target. Figure $1 \mathrm{~b}$ ) shows results of radiation-hydrodynamic simulations using the code HELIOS [26] indicating homogeneous mass density $\left(\rho=3.8 \mathrm{~g} / \mathrm{cm}^{3}\right)$ and pressure $(p=1.8 \mathrm{Mbar})$ profiles at the end of the drive. The electron temperature rises from $k_{\mathrm{B}} T_{\mathrm{e}}=0.2 \mathrm{eV}$ in the shock front to approximately $1 \mathrm{eV}$ at $10 \mu \mathrm{m}$ behind the drive side.

Ultrashort pulse laser produced K- $\alpha$ radiation probes the compressed boron plasma by x-ray Thomson scat- tering. Energies of $250 \mathrm{~J}$ are delivered at a pulse duration of $10 \mathrm{ps}$ and at $1053 \mathrm{~nm}$ wavelength, yielding intensities of $\approx 10^{17} \mathrm{~W} / \mathrm{cm}^{2}$. Vanadium and copper foils of $10 \mu \mathrm{m}$ thickness provide $\mathrm{K}-\alpha$ photon energies of $4.95 \mathrm{keV}$ and $8.05 \mathrm{keV}$ respectively, with $k$-vectors between $k=$ $1.3 \AA^{-1}$ and $k=4.3 \AA^{-1}$, on separate target experiments.

$\mathrm{X}$-rays scattered by the dense plasma disperse in a cylindrically bent $70 \times 25 \mathrm{~mm}^{2}$ large graphite crystal (highly-oriented pyrolitic graphite) and are focused in a von Hamos setup onto an imaging plate detector (Fujifilm). A $100 \mu \mathrm{m}$ thick gold shield blocks the direct view of the spectrometer crystal to the intense x-ray probe source. The gold shield has been coated with several $\mu \mathrm{m}$ $\mathrm{CH}$ to avoid $\mathrm{Au}$ emission induced by stray light.

Figure 2 shows experimental and theoretical plasmon scattering data from shock-compressed B. The data at small momentum transfer show a strong plasmon resonance downshifted by $32 \mathrm{eV}$ from the $\mathrm{K}-\alpha$ probe energy of $4.95 \mathrm{keV}$, see Fig. 2 (a). This feature is one order of magnitude stronger compared to scatter from uncompressed targets, indicating the presence of a large fraction of free electrons due to shock induced ionization, which is consistent with earlier compression experiments using the diamond anvil-cell technique [27]. With increasing momentum transfer $k$ the plasmon is increasingly damped, and in the proximity of the Fermi momentum the plasmon energy is constant Fig. 2 (b) These observations agree with calculations for 1.3 times compressed B at ionization stage $Z_{f}=2.3$ corresponding to an electron density of $n_{\mathrm{e}}=4 \times 10^{23} \mathrm{~cm}^{-3}$.

The calculations apply the newly developed BMA+LFC model for the dynamical structure factor $[28]$,

$$
\begin{aligned}
& S_{\mathrm{ee}}(k, \omega)=\frac{\hbar k^{2}}{\pi m_{\mathrm{e}} \omega_{\mathrm{pl}}^{2}} n_{\mathrm{B}}(\omega) \times \\
& \operatorname{Im}\left[\frac{z}{i \nu(\omega)}\left(\frac{\chi_{\mathrm{e}}^{\mathrm{OCP}}(k, z) \chi_{\mathrm{e}}^{\mathrm{OCP}}(k, 0)}{\chi_{\mathrm{e}}^{\mathrm{OCP}}(k, z)+\frac{\omega}{i \nu(\omega)} \chi_{\mathrm{e}}^{\mathrm{OCP}}(k, 0)}\right)\right],
\end{aligned}
$$

where $\omega_{\mathrm{pl}}=\sqrt{n_{\mathrm{e}} e^{2} / \epsilon_{0} m_{\mathrm{e}}}$ is the plasma frequency, and $n_{\mathrm{B}}(\omega)$ is the Bose function. Furthermore $z=\omega+i \nu(\omega)$ with $\nu(\omega)$ being the complex valued collision frequency. The latter is calculated within second order perturbation theory (Born approximation) and in the long wavelength limit, $k \rightarrow 0$ [25]. At finite transfer momentum, also electron-electron interactions have to be considered. Their influence on the dynamical structure factor is described by the local field correction (LFC) $G(k)$ in the electron one-component plasma response function,

$$
\chi_{\mathrm{e}}^{\mathrm{OCP}}(k, \omega)=\frac{\chi_{\mathrm{e}}^{\mathrm{RPA}}(k, \omega)}{1-V(k)[1-G(k)] \chi_{\mathrm{e}}^{\mathrm{RPA}}(k, \omega)} .
$$

$\chi_{\mathrm{e}}^{\mathrm{RPA}}(k, \omega)$ is the electronic RPA response function, see e.g. Ref. [29], $V(k)=e^{2} / \varepsilon_{0} k^{2}$ is the Coulomb potential 


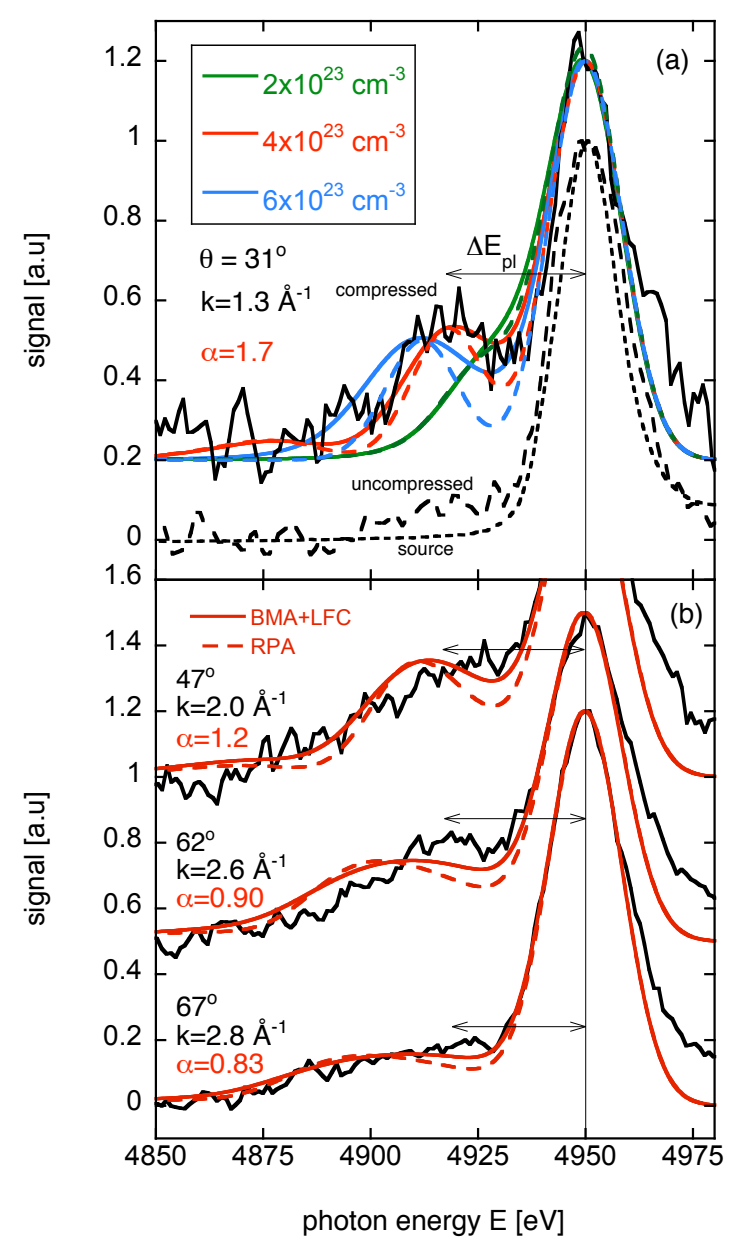

FIG. 2: (Color online) (a) X-ray scattering spectra (solid black) from compressed targets at $31^{\circ}$ scattering angle compared to fit calculations using the BMA+LFC model (solid colored curves) and the RPA (dashed colored curves) for three different densities. Also shown are the $\mathrm{V}$ probe spectrum (dotted) and scattering from uncompressed targets (black dashed).

(b) Scattering spectra for scattering angles $47^{\circ}, 62^{\circ}$, and $67^{\circ}$ and fit calculations using the BMA+LFC model (solid) and RPA (dashed). The experimental plasmon shift $\Delta E(k)$ for each angle is indicated by arrows.

in wavenumber representation. For the LFC, we use the parametrization by Farid et al. [30], supported by MonteCarlo simulations of dense electron liquids [31].

Roots in the denominator of Eq. (2) give rise to the plasmon resonances observed in the scattering spectrum. The LFC factor being a positive valued quantity leads to a decrease in the plasmon resonance position. The LFC factor scales as $k^{2}$ at wavenumbers $k \ll k_{\mathrm{F}}, k_{\mathrm{F}}=$ $\left(3 \pi^{2} n_{\mathrm{e}}\right)^{1 / 3}$ being the Fermi wavenumber. Consequently, for conditions of Fig. 2 a) the plasmon energy shift is only weakly influenced by the LFC. In this limit the plasmon energy shift in BMA+LFC agrees with the values obtained in the RPA.

Figure 2 a shows calculations for BMA+LFC and RPA for three electron densities indicating sensitivity of the plasmon shift to the electron density. The plasmon shift, $\Delta E_{\mathrm{pl}}=(32 \pm 3) \mathrm{eV}$, yields the electron density $n_{\mathrm{e}}=$ $(4.0 \pm 0.7) \times 10^{23} \mathrm{~cm}^{-3}$.

Figure 2 (b) shows that the experimental plasmon shift (indicated by arrows in the figure) is constant within an error bar of $5 \mathrm{eV}$. This behavior is consis- rs tent with the BMA+LFC calculations and indicate increasing local field effects due to strong electron coupling $\left(\Gamma \simeq 1.4 r_{s}=2.5, r_{s}=\left[3 / 4 \pi n_{\mathrm{e}}\right]^{1 / 3} / a_{\mathrm{B}}\right.$ is the Brueckner parameter) [32]. Furthermore, due to low electron temperatures $k_{\mathrm{B}} T_{\mathrm{e}} \simeq 0.2 \mathrm{eV}$, we deal with a Fermi degenerate plasma, $k_{\mathrm{B}} T_{\mathrm{e}} / E_{\mathrm{F}}=0.01$, the Fermi energy is $E_{\mathrm{F}}=\hbar^{2} k_{\mathrm{F}}^{2} / 2 m_{\mathrm{e}}=19.8 \mathrm{eV}$. In this regime, the plasmon spectrum is not sensitive to $T_{\mathrm{e}}$.

Figure 3 (a) shows the experimental plasmon shift as a function of momentum transfer $k$ compared to the RPA and BMA calculations both with and without local field corrections. In the limit $k \rightarrow 0$, all three models converge towards the plasma frequency, in agreement with the data. With increasing $k$ the experimental dispersion is constant at $\Delta E(k) \simeq 33 \mathrm{eV}$. The plasmon group velocity $v_{G}=d \omega(k) / d k$ vanishes, i.e. plasmons do not propagate at this energy. This behavior is in good agreement with the fully collisional BMA+LFC calculation but contradicting the RPA as well as the usual BMA that neglects the LFC factor [19].

In the strongly non-collective regime, the BMA+LFC calculation quickly converges into the single particle dispersion (Compton shift $\Delta E_{\text {Compton }}=\hbar^{2} k^{2} / 2 m_{\mathrm{e}}$ ), as expected and consistent with the RPA and the usual BMA. In this limit correlations become less important since distances smaller than the screening length are probed. Within the error margins, all three models are consistent with the non-collective scattering data at $k=4.3 \AA^{-1}$.

Finally, we analyse the plasmon width (rms width) as a function of $k$, results are shown in Fig. 3 (b) and compared to RPA and BMA with and without local field corrections. Instrumental and source broadening (c.f. the source spectrum in Fig. 2) have been subtracted. The two points at $k=1.3 \AA^{-1}$ and $2.0 \AA^{-1}$ corresponding to collective scattering show that plasmon damping is mainly due to electron-ion collisions. Conversely, the RPA severely underestimates the plasmon width.

At the present density, the critical wavenumber up to which plasmons are a well-defined mode is $k_{\mathrm{c}}=1.7 \AA^{-1}$. Above $k_{c}$, the plasmon merges with the single-pair continuum [18] giving rise to Landau damping in the RPA theory. At $k=1.3 \AA^{-1}$, we find the plasmon resonance at $E_{\mathrm{pl}}=32 \mathrm{eV}$, which lies beyond the cut-off energy of the single-pair continuum $\hbar \omega_{\text {pair }} \leq \hbar^{2}\left(k^{2}+2 k k_{\mathrm{F}}\right) / 2 m_{\mathrm{e}}=$ 


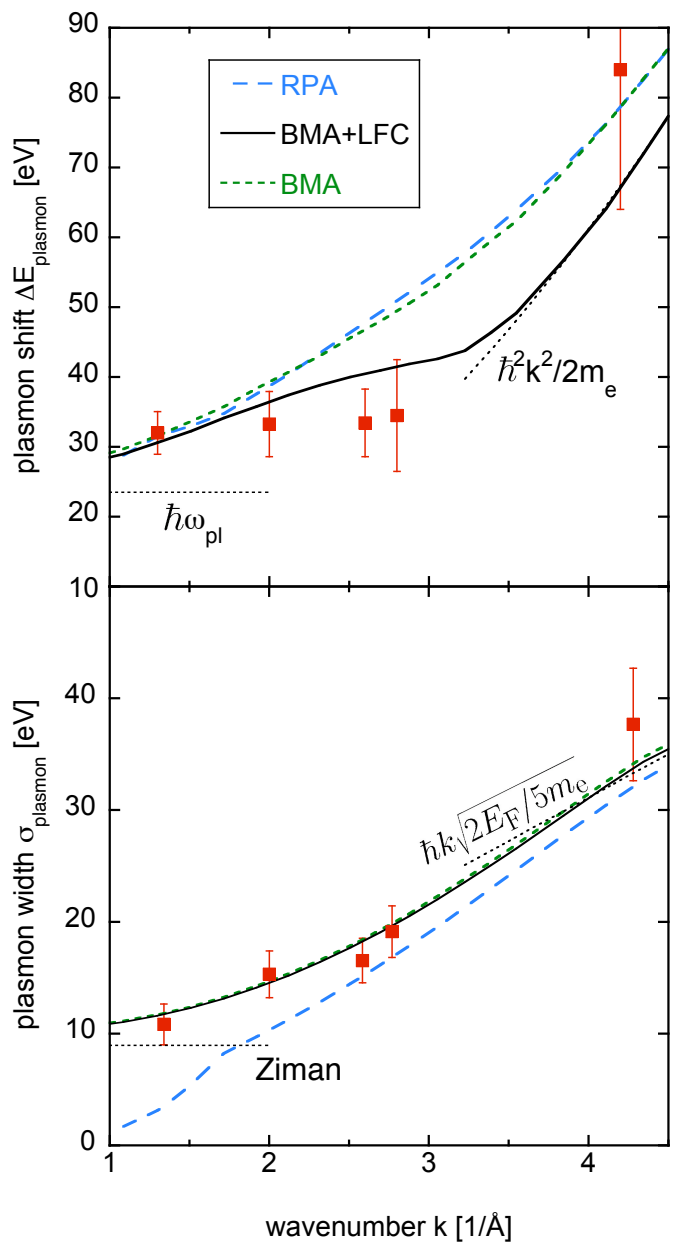

FIG. 3: (Color online) Plasmon dispersion (a) and rms-width (b) as function of the scattering wavevector $k$. Experimental data are compared to RPA (blue dashed), BMA with LFC (solid black) and BMA without LFC (green small dashes) calculations. The black dotted lines represent the limits of dispersion and damping at small and large $k$, respectively.

$29.1 \mathrm{eV}$. The sharp edge of the pair continuum reflects the steplike Fermi distribution of degenerate electrons. Hence, within the RPA, the plasmon mode cannot couple to the single-pair continuum and therefore is undamped.

At $47^{\circ}$ scattering angle, $k=2.0 \AA^{-1}$, the plasmon appears at $E_{\mathrm{pl}}=33 \mathrm{eV}$, which is within the single-pair continuum $\hbar \omega_{\text {pair }} \leq 50.1 \mathrm{eV}$. Hence, Landau damping is finite, explaining the increased spectral width in the RPA calculation as compared to the $31^{\circ}$ case. However, RPA still underestimates the plasmon damping, whereas we obtain good agreement using the BMA + LFC model.

We also show the Ziman result [33] as the asymptotic limit of collisional damping as $k \rightarrow 0$. In the Compton scattering regime $(\alpha<1)$, where the width approaches that of the Compton profile $\sigma_{\text {Compton }}=\hbar k \sqrt{2 E_{\mathrm{F}} / 5 m_{\mathrm{e}}}$ both RPA and BMA agree and are consistent with the data.

In conclusion, we demonstrated $k$-vector and frequency resolved x-ray Thomson scattering on plasmons in shockcompressed matter. Our data show very good agreement with the fully collisional BMA+LFC model for the dynamical structure factor. The calculations explain the plasmon broadening due to electron-ion collisions as well as vanishing plasmon dispersion due to strong electron-electron coupling. In the limits of small and large $k$-vectors, we observe the well-known analytical limits for the plasmon dispersion and damping.

This work was performed under the auspices of the U.S. Department of Energy by Lawrence Livermore National Laboratory under Contract DE-AC52-07NA27344. This work was further supported by LDRD grant 08-ERI002. C.F. and S.H.G. also acknowledge support by the Alexander von Humboldt-Foundation.

[1] G. Chabrier, Plasma Phys. Controlled Fusion 51, 124014 (2009).

[2] N. Nettelmann, et al., Astrophys. J. 683, 1217 (2008).

[3] A. Burrows et al., Rev. Mod. Phys. 65, 301 (1993).

[4] A. Burrows, et al., Astrophys. J. 345, 939 (1989).

[5] D. G. Hicks, et al., Phys. Rev. B 79, 014112 (2009).

[6] M. Koenig, et al., Plasma Phys. Controlled Fusion 47, B441 (2005).

[7] P. M. Nilson, et al., Phys. Rev. E 79, 016406 (2009).

[8] J. Lindl et al., Phys. Plasmas 11, 339 (2004).

[9] M. D. Knudson, et al., Science 322, 1822 (2008).

[10] D. K. Bradley, et al., Phys. Rev. Lett. 102, 075503 (2009).

[11] A. L. Kritcher, et al., Science 322, 69 (2008).

[12] V. E. Fortov, et al., Phys. Rev. Lett. 99, 185001 (2007).

[13] S. H. Glenzer et al., Rev. Mod. Phys. 81, 1625 (2009).

[14] S. H. Glenzer, et al., Phys. Rev. Lett. 98, 065002 (2007).

[15] R. Redmer, et al., IEEE Trans. Plasma Sc. 33, 77 (2005).

[16] C. Fortmann, et al., Laser Part. Beams 27, 311 (2009).

[17] H. J. Lee, et al., Phys. Rev. Lett. 102, 115001 (2009).

[18] D. Pines et al., The Theory of Quantum Liquids, vol. 1 (Benjamin, New York, 1966).

[19] R. Thiele, et al., Phys. Rev. E 78, 026411 (2008).

[20] D. Bohm et al., Phys. Rev. 75, 1851 (1949).

[21] W. Schülke, et al., Phys. Rev. B 33, 6744 (1986).

[22] W. Schülke, et al., Phys. Rev. B 54, 17464 (1996).

[23] B. C. Larson, et al., Phys. Rev. Lett. 77, 1346 (1996).

[24] K. Utsumi et al., Phys. Rev. B 23, 3291 (1981).

[25] H. Reinholz, et al., Phys. Rev. E 62, 5648 (2000).

[26] J. J. MacFarlane, et al., J. Quant. Spectrosc. Radiat. Transfer 99 (2006).

[27] M. I. Eremets et al., Science 293, 272 (2001).

[28] C. Fortmann, et al., Phys. Rev. E 81, 026405 (2010).

[29] N. R. Arista et al., Phys. Rev. A 29, 1471 (1984).

[30] B. Farid, et al., Phys. Rev. B 48, 11602 (1993).

[31] S. Moroni, et al., Phys. Rev. Lett. 75, 689 (1995).

[32] In Fermi degenerate systems, the coupling parameter scales as $1 / E_{\mathrm{F}} r_{s} \propto r_{s}$, instead of $1 / T r_{s}$ as in classical 
plasmas.

[33] G. Röpke, et al., Phys. Rev. E 60, R2484 (1999). 